\title{
BMJ Open Does specialist review for patients with suspected heart failure predict better outcomes? An observational study on the utility of compliance with NICE guidelines
}

Geraint Morton, Legate Philip, Thomas Gilpin, Pik Ee Chan, Kaushik Guha, Paul R Kalra

To cite: Morton G, Philip L, Gilpin T, et al. Does specialist review for patients with suspected heart failure predict better outcomes? An observational study on the utility of compliance with NICE guidelines. BMJ Open 2018;8:e021856. doi:10.1136/ bmjopen-2018-021856

\section{- Prepublication history for} this paper is available online. To view these files, please visit the journal online (http://dx.doi. org/10.1136/bmjopen-2018021856).

Received 21 January 2018 Revised 8 May 2018 Accepted 25 July 2018
Check for updates

(C) Author(s) (or their employer(s)) 2018. Re-use permitted under CC BY-NC. No commercial re-use. See rights and permissions. Published by BMJ.

Department of Cardiology, Queen Alexandra Hospital, Portsmouth Hospitals NHS Trust, Portsmouth, Wessex, UK

Correspondence to Dr Geraint Morton; geraintmorton@gmail.com

\section{ABSTRACT}

Objectives Compare outcomes in patients with suspected heart failure (HF) and raised natriuretic peptides who are reviewed in a specialist HF clinic in line with National Institute for Health and Care Excellence (NICE) guidelines (compliant group) versus patients who are not reviewed in the clinic (non-compliant group).

Design Retrospective observational study.

Setting Single large UK district general hospital.

Participants 567 consecutive patients in primary care with raised $\mathrm{N}$-terminal pro-brain natriuretic peptide (NTpro-BNP) levels (>400 pg/mL) from February to September 2014.

Interventions 161 (28\%) patients were referred to the specialist HF clinic and 406 (72\%) were not. Outcomes were compared between the two groups.

Outcome measures All-cause and cardiovascular (CV) hospitalisations and all-cause mortality.

Results The compliant group were slightly younger than the non-compliant group ( $78 \pm 9$ vs $80 \pm 9 ; p=0.019$ ) but had much higher NT-pro-BNP $(3108 \pm 4526$ vs $2271 \pm 3637 \mathrm{pg} / \mathrm{mL} ; \mathrm{p}<0.0001)$. Despite this, over a mean follow-up period of $9 \pm 2$ months, rates of allcause hospitalisation $(24 \%$ vs $44 \%$; $p<0.0001)$ and CV hospitalisation ( $3 \%$ vs $15 \%, p<0.0001)$ were significantly lower in the compliant group versus the non-compliant group, respectively. There was no significant difference in mortality rates $(6 \%$ compliant group vs $8 \%$ non-compliant group; $p=0.487$ ).

Conclusions Hospitalisation rates in patients with suspected HF and raised NT-pro-BNP were extremely high over a relatively short follow-up period. Patients reviewed in a specialist HF clinic had much higher NTpro-BNP levels, suggesting they were at higher risk of adverse outcomes, yet also had significantly lower rates of all-cause and CV hospitalisation. Our findings support implementation of the relevant NICE guidelines for patients with suspected HF.

\section{INTRODUCTION}

Heart failure (HF) is a common, severe, chronic disease. It causes significant

\section{Strengths and limitations of this study}

- There are few data supporting the 2 and 6 weeks waiting-time targets for specialist assessment recommended by National Institute for Health and Care Excellence (NICE) for patients in primary care with suspected heart failure.

- This study examines whether adhering to the NICE guidelines is associated with better patient outcomes and therefore provides some insight into the potential benefits of guideline implementation.

- The data are observational and therefore causation and association cannot be fully separated.

morbidity and has a poor prognosis characterised by multiple hospital admissions and death with associated implications both for patients' quality of life and resources. In the UK, approximately 900000 people are affected. ${ }^{1} \mathrm{HF}$ accounts for around $5 \%$ of all emergency medical admissions and $2 \%$ of all National Health Service (NHS) inpatient bed-days. ${ }^{2}$ Despite the availability of evidencebased treatments which improve prognosis (particularly for patients with HF and reduced ejection fraction, HFREF) contemporary data demonstrate outcomes in acute and chronic HF that still compare unfavourably to many cancers. ${ }^{2-4}$ In recognition of these poor outcomes, the UK's National Institute for Health and Care Excellence (NICE) introduced chronic HF guidelines (CG108) ${ }^{2}$ and quality standards ${ }^{5}$ to improve HF care. These guidelines recommend that patients with suspected HF are referred for a timely diagnosis through specialist assessment and transthoracic echocardiography. A specialist is defined as 'a doctor with subspecialty interest in HF (often a consultant cardiologist) who leads a specialist multidisciplinary 
HF team of professionals'. Those with a history of prior myocardial infarction or very high natriuretic peptide levels $(\mathrm{BNP}>400 \mathrm{pg} / \mathrm{mL}$ or N-terminal pro-brain natriuretic peptide [NT-pro-BNP] $>2000 \mathrm{pg} / \mathrm{mL}$ ) should be seen within 2 weeks and patients with more modest elevations in natriuretic peptides (BNP $>100 \mathrm{pg} / \mathrm{mL}$ or NT-pro-BNP $>400 \mathrm{pg} / \mathrm{mL}$ ) should be seen within 6 weeks. Patients with natriuretic peptide levels below these thresholds should be investigated for alternative diagnoses. While specialist care for patients with HF has been repeatedly shown to improve patient outcomes ${ }^{67}$ there are a paucity of data to support this waiting time-based referral pathway which is modelled on similar NICE referral pathways for patients with suspected cancer. ${ }^{8}$ Moreover, this cancer waiting time target is itself arbitrary and it is unclear whether adherence to it results in better outcomes. ${ }^{9}$

Following the publication of NICE chronic HF guidelines, a dedicated one-stop, fully NICE compliant referral pathway and specialist clinic was established at our institution to assess patients with suspected HF. The clinic launch was accompanied by education of referring general practitioners (GPs) and provision of NT-pro-BNP assays. Despite this, not all patients with suspected HF were referred to secondary care but were managed in non-specialist primary care settings, and thus not in compliance with guidelines. Our aim was to compare outcomes in patients with suspected HF referred for specialist diagnosis and management in accordance with NICE guidelines to those who were not.

\section{METHODS}

This was a single-centre, retrospective evaluation of outcomes in patients with suspected HF conducted at Portsmouth Hospitals NHS Trust, a very large district general hospital serving a population of 675000 on the South coast of England. Portsmouth Hospitals serves a predominantly white population $(>90 \%$ in UK Census data) who live in both urban and rural environments. According to Public Health England and the Office for National Statistics, the urban population is characterised by high levels of social deprivation, poor health and a below UK average life expectancy. In contrast the rural population has relatively low levels of social deprivation, better health and above UK average life expectancy.

A dedicated pathway was established for assessment of patients with suspected HF in keeping with NICE guidelines. Information on the referral pathway and its appropriate use was disseminated to all primary care referrers. NT-pro-BNP assays were made available in primary care solely for use in patients with suspected new diagnoses of HF. Referrals to the specialist HF clinic were sent via a dedicated fax number. Patients referred with an NT-pro-BNP of $>2000 \mathrm{pg} / \mathrm{mL}$ were seen within 2 weeks and those with an NT-pro-BNP of 400-2000 pg/mL within 6 weeks. Participants were seen in a one-stop clinic and received specialist assessment and echocardiography. A diagnosis of HF could then be confirmed or excluded, and an appropriate management plan formulated. Patients with HF were managed in line with the European Society of Cardiology (ESC) HF guidelines ${ }^{10}$ wherever possible and appropriate. Those with HFREF routinely received early and ongoing management from a multidisciplinary team centred around community-based HF nurse specialists and supported by HF specialists. Those with a diagnosis of HFPEF and those without HF were usually discharged back to the GP with a management plan.

All patients with a raised NT-pro-BNP sample processed at our laboratory during the first 8 months of the specialist HF clinic operating (February to September 2014) were identified. The biochemistry laboratory at the hospital is the central laboratory that processes all the samples from the GP practices in the surrounding area. Serum samples were processed using a Biomerieux sandwich immunoassay which measures NT-pro-BNP over a range of $15-25000 \mathrm{pg} / \mathrm{mL}$. Participants were divided into two groups: those referred to the HF clinic (compliant group) and those who were not referred to the clinic (non-compliant group). Hospital coding data, information services, electronic records and case notes were used to identify hospital admissions, reason for admission and deaths in all patients over a minimum follow-up period of 6 months from the time of the raised NT-pro-BNP result. Individual case note assessment was performed if further information was required. Comparisons were then made between the two cohorts.

Secondary comparisons in hospitalisation and mortality rates were made within NICE compliant group between those whose assessment was delayed and those whose assessment was not delayed. In patients with an NT-pro-BNP of 400-2000 pg/mL, a delay was defined as either an interval between the positive blood test result and referral of $>10$ days or between referral and a clinic appointment date offer of $>6$ weeks. In patients with an NT-pro-BNP of $>2000 \mathrm{pg} / \mathrm{mL}$, this was defined as $>5$ days or 2 weeks, respectively.

Microsoft Excel with XLSTAT software was used for all statistical analyses. Data are presented as mean \pm SD. For continuous variables, Shapiro-Wilk analyses checked normality of the underlying distribution. Having determined that non-parametric tests were required the two groups were compared using either the Mann-Whitney U test or the Fisher's exact test for binomial data. Significance was determined if two-sided $\mathrm{p}$ values were $<0.05$.

\section{Patient and public involvement}

Patients and the public were not directly involved in the study development, design, recruitment or conduct. The results will be disseminated to primary care locally.

\section{RESULTS}

\section{Patient population}

Five hundred and sixty-seven consecutive patients with a raised NT-pro-BNP were identified and included: 161 
Table 1 Baseline characteristics

\begin{tabular}{lllc}
\hline & $\begin{array}{l}\text { Compliant } \\
\mathbf{n = 1 6 1}\end{array}$ & $\begin{array}{l}\text { Non- } \\
\text { compliant } \\
\mathbf{n = 4 0 6}\end{array}$ & P values \\
\hline Age & $78 \pm 9$ & $80 \pm 9$ & 0.019 \\
\hline NT-pro-BNP $(\mathrm{pg} / \mathrm{mL})$ & $3108 \pm 4526$ & $2271 \pm 3637$ & $<0.0001$ \\
Male & $78(48 \%)$ & $194(48 \%)$ & 0.926 \\
\hline NT-pro- & $68(42 \%)$ & $118(29 \%)$ & \\
BNP >2000 pg/mL & & & 0.003 \\
Haemoglobin $(\mathrm{g} / \mathrm{dL})$ & $12.8 \pm 20$ & $12.5 \pm 18$ & 0.151 \\
Creatinine $(\mu \mathrm{mol} / \mathrm{L})$ & $102 \pm 54$ & $107 \pm 65$ & 0.418 \\
\hline
\end{tabular}

$(28 \%)$ in the compliant group and $406(72 \%)$ in the non-compliant group. The mean follow-up period was $9 \pm 2$ months.

The compliant group patients were slightly younger and had much higher NT-pro-BNP than the non-compliant group. Baseline characteristics of the patients are shown in table 1 .

Haemoglobin/creatinine data were available within 3 months of NT-pro-BNP sample for $98 \% / 98 \%$ of the compliant group and $95 \% / 99 \%$ of the non-compliant group.

Eighty-five $(53 \%)$ compliant patients were confirmed to have $\mathrm{HF}$ after echocardiography and specialist assessment. Of those with HF, 39 (46\%) had HFREF and 46 $(54 \%)$ had HFPEF. Of 161, $100(62 \%)$ had hypertension, $84(52 \%)$ atrial fibrillation and $32(20 \%)$ diabetes.

\section{Patient outcomes}

Compliant group patients were much less likely to be hospitalised than non-compliant patients. All-cause hospitalisation rates were $39 / 161$ (24\%) vs $180 / 406$ (44\%); $<<0.0001$ for the compliant and non-compliant group, respectively. Cardiovascular (CV) admissions were also much lower in the compliant group; $5 / 161$ (3\%) vs

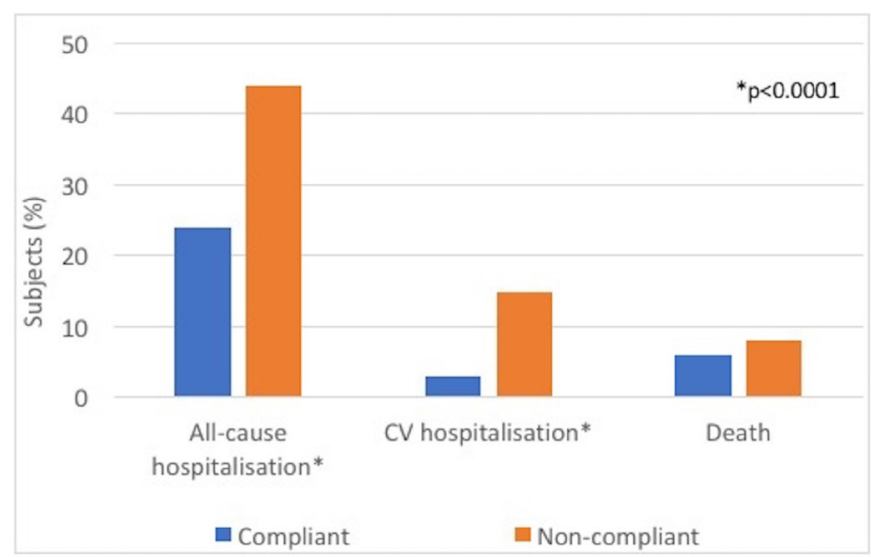

Figure 1 Outcomes for patients with suspected HF during a mean follow-up of 9 months according to whether they were compliant with NICE recommendations to refer for specialist evaluation. CV, cardiovascular; HF, heart failure; NICE, National Institute for Health and Care Excellence.
Table 2 Number of patients on the 2 and 6 weeks pathways and intervals between a positive NT-pro-BNP and referral, and referral and clinic review

\begin{tabular}{|c|c|c|}
\hline & 2 weeks & 6 weeks \\
\hline Patients, n (\%) & $68(42)$ & 93 (58) \\
\hline $\begin{array}{l}\text { Offered review within target, } \\
\mathrm{n}(\%)\end{array}$ & 67 (99) & $92(99)$ \\
\hline $\begin{array}{l}\text { Interval between NT-pro-BNP } \\
\text { and referral (days) }\end{array}$ & $13 \pm 12$ & $19 \pm 17$ \\
\hline $\begin{array}{l}\text { Interval between referral and } \\
\text { clinic review (days) }\end{array}$ & $10 \pm 5$ & $24 \pm 12$ \\
\hline
\end{tabular}

$59 / 406(15 \%), \mathrm{p}<0.0001$. There was a non-significant trend towards lower mortality rates in the compliant group; $10 / 161(6 \%)$ vs $34 / 406(8 \%), p=0.487$. Outcome results are summarised in figure 1.

\section{NICE compliant patient pathway}

Of 161, 68 (42\%) of patients had an NT-pro-BNP > 2000 pg/ $\mathrm{mL}$ and were on the 2-week pathway. Of 68, 67 (99\%) were offered an appointment within the 2-week target. Four of these patients declined the first appointment offered, therefore, overall 63/68 (93\%) were seen within 2 weeks (table 2). Of 93, 92 (99\%) patients on the 6-week pathway were offered and accepted an appointment within 6 weeks. Three $(2 \%)$ patients were admitted to hospital after the positive NT-pro-BNP result but before they were due to be reviewed in clinic but were still included as part of the compliant group. They included $2(3 \%)$ patients on the 2-week pathway and $1(1 \%)$ patient on the 6 -week pathway. One of these patients, who was on the 2-week pathway, died during that admission.

One hundred and sixteen $(72 \%)$ patients experienced delays in the pathway. Mean NT-pro-BNP in those patients who were delayed was $3521 \pm 5138 \mathrm{pg} / \mathrm{mL}$ compared with $2043 \pm 1785 \mathrm{pg} / \mathrm{mL}$ in those who were not. Delays in the pathway were not associated with a worse outcome. There were no differences in all-cause hospitalisations: $12(26 \%)$ vs 27 (23\%), p=0.68; CV admissions: 2 (4\%) vs $3(3 \%)$, $\mathrm{p}=0.619$ or deaths: $1(2 \%)$ vs $9(8 \%), \mathrm{p}=0.285$, for those not delayed versus those delayed, respectively.

\section{DISCUSSION}

This service evaluation demonstrates that patients with suspected $\mathrm{HF}$ and raised natriuretic peptides have a very high rate of adverse outcomes in the short term. Timely HF specialist involvement is associated with better outcomes, specifically lower rates of hospitalisation. These novel, real-world data support the implementation of HF pathways which comply with NICE guidelines.

The main strengths of this work are that this is a systematic evaluation of consecutive real-world patients with suspected HF and that those referred to specialist services received guideline-based treatment delivered by an established comprehensive HF service. It also provides valuable 
contemporary data from a cohort representative of a very large population of patients with poor outcomes who remain understudied. Furthermore, while NICE recommendations on referral pathways were produced because of the adverse outcomes in this population, the waiting-time targets predate relevant supporting evidence. The main limitation of the data is that they are retrospective and observational and therefore demonstrate an association rather than clear causation. There will be some unadjusted confounding factors and the possibility of referral bias influencing outcomes. However, while the non-compliant group were slightly older, the compliant group had much higher NT-pro-BNP levels, which itself is independently associated with a higher risk of adverse outcomes. ${ }^{11}$ The much lower hospitalisation rates seen in the compliant group, therefore, suggest that there is a real benefit in being seen in a specialist HF clinic.

These findings are consistent with the substantial body of evidence demonstrating that outcomes for patients with $\mathrm{HF}$ are improved within appropriate systems of care. ${ }^{6}$ Specialist multidisciplinary care reduces both all-cause and HF hospitalisation. ${ }^{7}$ Furthermore, in contrast to many drug and device therapies, the literature suggests that all patients with HF, including those with HFPEF, benefit from specialist care. Again, our data are consistent with this in that we included all patients regardless of left ventricular ejection fraction. The lower rates of hospitalisation observed in the compliant patients are likely to be due, at least in part, to specialist care. Expert care involves accurate diagnosis and management including appropriate use of therapies which improve outcomes. Given the short follow-up period of this study, drug therapies will have been the most important interventions and have previously been well documented to reduce hospitalisations over short follow-up periods for patients with HFREF. ${ }^{10}$ An additional benefit for compliant patients with $\mathrm{HF}$ and in particular those with HFREF in this study is likely to relate to ongoing access to the multidisciplinary team. Patients in the non-compliant group would have been much less likely to be managed by the multidisciplinary team who receive the vast majority of new referrals from specialists.

Since raised natriuretic peptides are known to predict an adverse outcome in patients without overt HF as well as in those with $\mathrm{HF},{ }^{11}{ }^{12}$ it is likely that better outcomes in the compliant group are not solely due to better management of HF. For example, HF physicians are also well placed to manage other CV diseases, such as atrial fibrillation, which can mimic HF and causes elevations in natriuretic peptides. We did not individually evaluate the causes for each admission, however, it seems likely that early assessment by a physician led to changes in management for patients without HF in addition to those with HF. Similarly, it is also of interest that all-cause hospitalisations were lower and not just CV hospitalisations. This may in part be explained by the fact that HF causes multisystem adverse effects. A proportion of the observed hospitalisations may have been due to sequelae of $\mathrm{HF}$ which are not obviously CV, such as renal impairment. Furthermore, HF hospitalisations may not always be recorded as such. For example, while patients presenting with oedema due to HF may be recorded as an HF hospitalisation, they may also be recorded as being hospitalised due to fluid overload a non-CV cause. Misclassification of some causes of hospitalisation may be more likely as non-clinical administrative staff record hospitalisation causes in our institution, a practice which is the norm in the UK.

The low referral rate $(28 \%)$ of patients with raised natriuretic peptides is noteworthy. More detailed data on those patients who were not referred are not available. However, the local healthcare provision is organised such that our centre provides the vast majority of secondary care, and thus specialist services including cardiology and HF. A small minority of patients may have been referred elsewhere, such as to the private sector. However, it can be reasonably assumed that most of the non-compliant patients were managed in primary care by their GP. As the HF pathway was established shortly before this evaluation commenced some GPs may not have known about the service despite our efforts to raise awareness and the incorporation of the referral pathway into their standard online resources. It is also likely that in many cases GPs underestimate the severity and complexity of HF and felt they could manage these patients themselves. Contemporary data suggest that GPs have relatively high levels of confidence in the diagnosis and management of $\mathrm{HF}^{13}$ Another reason for the relatively low referral rate may relate to GPs testing patients in the non-compliant group in clinical situations outwith the pathway. Our pathway specified that natriuretic peptide levels should only be checked for patients with a potential new diagnosis of HF in keeping with NICE guidelines. However, NT-pro-BNP may, in some cases, have been used to monitor patients with known HF or even in the absence of a clinical suspicion of HF. Some patients may also have chosen against referral but again this is likely to account for a small proportion of the non-compliant patients.

The purpose of including referral-time targets in NICE guidelines is so that patients with suspected HF are seen rapidly as they are at high risk of adverse outcome. Despite offering a service compliant with these targets, we identified notable delays between a positive NT-pro-BNP result in primary care and referral to clinic. For those on the 2-week pathway, the interval between a positive NT-pro-BNP and referral to the HF clinic was longer than the interval between receipt of the referral and patient review in clinic. This seems counterproductive and unnecessary in a time-critical pathway but was not associated with worse outcomes. The absence of worse outcomes in patients who were delayed may be due to the low numbers who did not experience a delay or because rapid specialist review compensated for what were relatively short delays experienced earlier in the pathway.

The true magnitude of the benefit of early specialist review for patients with suspected HF and raised natriuretic peptides needs to be clarified. The body of evidence 
supporting specialist care for patients with HF means that it would be difficult to justify a randomised controlled trial that would deliberately put patients at risk. However, it may be possible to compare outcomes in regions with no established NICE compliant services to other areas with established compliant services. Our findings are in keeping with the totality of the evidence and provide further data supporting specialist care. However, since it is a single-centre study, there may be centre specific effects at work and it is important to demonstrate that these findings can be reproduced in multiple centres. Subgroup analysis has been deliberately limited to prevent analysis of small groups and reduce the likelihood of spurious findings. As a result, the benefits of specialist review for particular patient subgroups, for example, those with HFREF versus HFPEF are not known from these data. The growing HF burden means that further research to build on this work and answer these questions should be considered an urgent priority.

\section{CONCLUSION}

This study confirms that real-world patients with suspected $\mathrm{HF}$ and raised natriuretic peptides have an adverse shortterm prognosis with high rates of hospitalisations and mortality. NICE chronic HF recommendations were created in recognition of this, however, data supporting the implementation of the diagnostic aspects of these guidelines have been lacking. Specialist care improves outcomes for all patients with $\mathrm{HF}$ and an early review for patients with suspected HF in a NICE compliant clinic is associated with reduced rates of all-cause hospitalisation. Centres offering a comprehensive HF service should set up pathways in line with NICE recommendations and GPs should be encouraged to refer to these services.

Contributors GM defined the methods, acquired, analysed and interpreted the data and drafted the manuscript. LP acquired and analysed the data and drafted the manuscript. TG, PC and KG helped acquire and analyse the data and critically revised the manuscript. PRK defined the methods, acquired and interpreted data and critically revised the manuscript. All authors read and approved the final manuscript.

Funding The authors have not declared a specific grant for this research from any funding agency in the public, commercial or not-for-profit sectors.
Competing interests None declared.

Patient consent Not required.

Ethics approval Formal ethical approval was not required, however, the study was registered with the Trust's Clinical Effectiveness Department.

Provenance and peer review Not commissioned; externally peer reviewed.

Data sharing statement No additional data are available.

Open access This is an open access article distributed in accordance with the Creative Commons Attribution Non Commercial (CC BY-NC 4.0) license, which permits others to distribute, remix, adapt, build upon this work non-commercially, and license their derivative works on different terms, provided the original work is properly cited, appropriate credit is given, any changes made indicated, and the use is non-commercial. See: http://creativecommons.org/licenses/by-nc/4.0/.

\section{REFERENCES}

1. Heart failure supplement. 2002 https://www.bhf.org.uk/publications/ statistics/heart-failure-supplement-2002 (accessed 29 Dec 2017).

2. NICE. Chronic heart failure in adults: management | Guidance and guidelines. https://www.nice.org.uk/guidance/cg108 (accessed 29 Dec 2017).

3. Maggioni AP, Dahlström U, Filippatos G, et al. EURObservational Research Programme: regional differences and 1-year follow-up results of the Heart Failure Pilot Survey (ESC-HF Pilot). Eur J Heart Fail 2013;15:808-17.

4. Askoxylakis V, Thieke C, Pleger ST, et al. Long-term survival of cancer patients compared to heart failure and stroke: a systematic review. BMC Cancer 2010;10:105.

5. NICE. Chronic heart failure in adults | Guidance and guidelines. https://www.nice.org.uk/guidance/qs9 (accessed 29 Dec 2017).

6. British society for heart failure. National heart failure audit. http:// www.ucl.ac.uk/nicor/audits/heartfailure/documents/annualreports/ annual-report-2015-6-v8.pdf (accessed 29 Dec 2017).

7. Morton G, Masters J, Cowburn PJ. Multidisciplinary team approach to heart failure management. Heart 2018;104:1376-82.

8. NICE. Suspected cancer: recognition and referral | Guidance and guidelines. https://www.nice.org.uk/guidance/ng12/chapter/1recommendations-organised-by-site-of-cancer (accessed $29 \mathrm{Dec}$ 2017).

9. Harrison AJ, Foot CS. Targets and prioritization: the case of cancer in the English NHS. Qual Prim Care 2012;20:125-9.

10. Ponikowski P, Voors AA, Anker SD, et al. 2016 ESC Guidelines for the diagnosis and treatment of acute and chronic heart failure: The Task Force for the diagnosis and treatment of acute and chronic heart failure of the European Society of Cardiology (ESC)Developed with the special contribution of the Heart Failure Association (HFA) of the ESC. Eur Heart J 2016;37:2129-200.

11. Wang TJ, Larson MG, Levy D, et al. Plasma natriuretic peptide levels and the risk of cardiovascular events and death. $N$ Engl J Med 2004;350:655-63.

12. Doust JA, Pietrzak E, Dobson A, et al. How well does B-type natriuretic peptide predict death and cardiac events in patients with heart failure: systematic review. BMJ 2005;330:625.

13. Hancock HC, Close $H$, Fuat $A$, et al. Barriers to accurate diagnosis and effective management of heart failure have not changed in the past 10 years: a qualitative study and national survey. BMJ Open 2014;4:e003866. 